\title{
Thank you to JARG's top reviewers
}

Published online: 21 June 2017

(C) Springer Science+Business Media, LLC 2017

A scientific journal's greatest responsibility is to ensure that all contributions accepted for publication are rigorously but fairly reviewed. We gratefully acknowledge the valued support of the following scientists who have contributed most frequently to our review process since January 2016. Your dedication is greatly appreciated!

Ronit Abir
Marwan Alhalabi
Richard Anderson
Georgios Anifandis
Asangla Ao
Valerie Baker
David Barad
Ana Karina Bartmann
Daniela Bebbere
Yaakov Bentov
Claire E. Beyer
Bianca Bianco
Gustavo Blanco
Jeffrey Boldt
Edson Borges Jr.
Paul R. Brezina
John Bromfield
Rita Canipari
Robert F. Casper
Tiencheng Arthur Chang
Hector Chemes
Ri-Cheng Chian
Indrajit Chowdhury
Lane Christenson
Danilo Cimadomo
Juan R. Correa-Perez
Carolyn Coulam
Brian Dale
Sarah Darmon
Maurizio Dattilo
Lucia De Santis

Ronit Abir

Georgios Anifandis

Asangla Ao

Ana Karina Bartmann

Daniela Bebbere

Yaakov Bentov

Gustavo

Jeffrey Boldt

Edson Borges Jr

Paul R. B

Robert F. Casper

Tiencheng Arthur Chang

Lane Christenson

Danilo Cimadomo

Juan R. Correa-Perez

Brian Dale

Maurizio Dattilo

Lucia De Santis

\author{
Michel De Vos \\ Marie-Madeleine Dolmans \\ Andrew Dorfmann \\ Anil K. Dubey \\ Thomas Ebner \\ Michael Eisenberg \\ Navid Esfandiari \\ Sandro C. Esteves \\ Guoping Fan \\ Eric J. Forman \\ Elpida Fragouli \\ Jason Franasiak \\ William E. Gibbons \\ Elizabeth Ginsburg \\ Susan Gitlin \\ Norbert Gleicher \\ Kara N. Goldman \\ Randi H. Goldman \\ Valeria A. Gomes \\ Katherine A. Green \\ Darren Karl Griffin \\ Ndeye-Aicha Gueye \\ Karl R. Hansen \\ Shu Hashimoto \\ Maria Belen Herrero \\ Hananel Holzer \\ Jim Hotaling \\ Karla Hutt \\ Hisataka Iwata \\ Sangita Kathleen Jindal \\ Caroline Juneau
}

Daniel J Kaser

Alyce C. Katayama

William G. Kearns

Levent Keskintepe

S. Samuel Kim

Yong Jin Kim

Rebecca Krisher

Vitaly A. Kushnir

Antonio La Marca

Graça Lopes

Alberto Maria Luciano

Ronit Machtinger

Clara Marin

Diego Marin

Caitlin Elizabeth Martin

Wellington P. Martins

Elizabeth McGee

Lynda K. McGinnis

Marie McLaughlin

Yves Jean-Remi Menezo

Deepak Modi

Manuela Monti

Dean Morbeck

Scott Morin

Mohammad Hosein Nasr-Esfahani

Paula A. Navarro

Stefania A. Nottola

Sergio Oehninger

Kutluk Oktay

Raoul Orvieto

Kelly Pagidas
George Patounakis

Alexsandra Fernandes Pereira

Nigel Pereira

John Petrozza

Raul Eduardo Piña-Aguilar

Kimball Osterloh Pomeroy

Alexander Michael Quaas

Catherine Racowsky

Denny Sakkas

Glenn Schattman

Mitchel C. Schiewe

Carla Schmitz

Emre Utku Seli

Chandra Shenoy

Sherman Silber

Amy E.T. Sparks

Judy E. Stern

Nao Suzuki

Jason E. Swain

Tyl Taylor

Evelyn Elizabeth Telfer

Alison Ting

Mark Umbarger

Andrea Weghofer

Teresa K. Woodruff

Shuo Xiao

Hakan Yarali

Yiping Zhan

Rebekah Zimmerman 